\title{
Absence of discontinuity in the exchange-correlation potential in the band-gap problem at finite temperature
}

\author{
Tai Kai Ng \\ Department of Physics, Massachusetts Institute of Technology, Massachusetts 02139
}

(Received 22 September 1988)

\begin{abstract}
It is shown that there exists no discontinuity in the $\mathbf{q}=\mathbf{0}$ component of the exchange-correlation potential in the band-gap problem in the finite-temperature density-functional theory. The physical implication of this result is pointed out.
\end{abstract}

It has been shown by Sham and Schlüter ${ }^{1}$ and by Perdew and Levy ${ }^{2}$ that there is a discontinuity $\Delta$ in the $q=0$ component of the exchange-correlation potential $V_{\mathrm{xc}}(\mathbf{r})$ of the Kohn-Sham (KS) theory ${ }^{3,4}$ as one crosses a band gap. The true semiconductor (or insulator) band gap is given by ${ }^{5,6}$

$$
E_{g}=\varepsilon_{g}^{\mathrm{KS}}+\Delta,
$$

where $\varepsilon_{g}^{\mathrm{KS}}$ is the band gap derived from the KS equations and

$$
\Delta=V_{\mathrm{xc}}^{+}(0)-V_{\mathrm{xc}}^{-}(0),
$$

where

$$
V_{\mathrm{xc}}^{+}(0)=\lim _{\eta \rightarrow 0} V_{\mathrm{xc}}^{N+\eta}(0)
$$

and

$$
V_{\mathrm{xc}}^{-}(0)=\lim _{\eta \rightarrow 0} V_{\mathrm{xc}}^{N-\eta}(0)
$$

are the $\mathbf{q}=\mathbf{0}$ components of the exchange-correlation potentials for the $(N+\eta)$ - and $(N-\eta)$-particle systems, respectively, when the $N$-particle system is the insulating state with a filled valence band and an empty conduction band. However, their results were based on the $T=0 \mathrm{~K}$ (where $T$ is the temperature) density-functional theory. We shall study the corresponding $T \neq 0 \mathrm{~K}$ theory in this comment, using the finite-temperature formulation of Mermin. $^{7}$

We shall consider a perfect crystal with spatially extended states. First we want to show that the exact chemical potential $\mu$ of the system at finite temperature is given by

$$
\mu=\mu^{\mathrm{KS}} \text {, }
$$

as in the zero-temperature case..$^{4,8}$ The proof procedures as follows.

Using the thermodynamic identity ${ }^{9}$

$$
\left[\frac{\partial \Omega}{\partial \mu}\right]_{V}=-N,
$$

where $\Omega=F-\mu N$ is the grand-canonical potential and $F$ is the Helmoltz free energy, it can be proved easily that

$$
\frac{1}{V}\left(\frac{\partial F}{\partial n}\right)_{V}=\mu,
$$

where $V$ is the volume and $n$ the mean electron density. Notice that these variations are performed under fixed external potentials. ${ }^{10}$

In the Kohn-Sham theory, the Helmholtz free energy is written as a sum of the following terms: ${ }^{3,4}$

$$
\begin{aligned}
F= & \sum_{i} f_{i} \varepsilon_{i}-\int V_{\mathrm{xc}}(\mathbf{r}) n(\mathbf{r}) d \mathbf{r}+\frac{1}{2} \iint \frac{n(\mathbf{r}) n\left(\mathbf{r}^{\prime}\right)}{\left|\mathbf{r}-\mathbf{r}^{\prime}\right|} d \mathbf{r} d \mathbf{r}^{\prime} \\
& +F_{\mathrm{xc}}[n(\mathbf{r})]+\frac{1}{\beta} \sum_{i}\left[f_{i} \ln f_{i}+\left(1-f_{i}\right)\right]
\end{aligned}
$$

where $\varepsilon_{i}$ 's are the eigenvalues of the $\mathrm{KS}$ equation, $F_{\mathrm{xc}}$ is the exchange-correlation part of the free energy, 3,4 $\beta=\left(k_{B} T\right)^{-1}, n(\mathbf{r})$ the electron density distribution, and

$$
f_{i}=\frac{1}{e^{\beta\left(\varepsilon_{i}-\mu^{\mathrm{KS}}\right)}+1}
$$

is the Fermi distribution function. Therefore, with Eq. (6), it is straightforward to obtain (see also Ref. 4)

$$
\begin{aligned}
\mu= & \mu^{\mathrm{KS}}+\left.\int \frac{\delta V^{\mathrm{KS}}(\mathbf{r})}{\delta n}\right|_{V} n(\mathbf{r}) d \mathbf{r}-\left.\int \frac{\delta V^{\mathrm{KS}}(\mathbf{r})}{\delta n}\right|_{V} n(\mathbf{r}) d \mathbf{r} \\
& +\left.\int V^{\mathrm{ext}}(\mathbf{r}) \frac{\delta n(\mathbf{r})}{\delta n}\right|_{V} d \mathbf{r}-\left.\int V^{\mathrm{KS}}(\mathbf{r}) \frac{\delta n(\mathbf{r})}{\delta n}\right|_{V} d \mathbf{r} \\
& +\left.\int \frac{\delta n(\mathbf{r})}{\delta n}\right|_{V} \frac{n\left(\mathbf{r}^{\prime}\right)}{\left|\mathbf{r}-\mathbf{r}^{\prime}\right|} d \mathbf{r} d \mathbf{r}^{\prime}+\left.\int \frac{\delta n(\mathbf{r})}{\delta n}\right|_{V} \frac{\delta F_{\mathrm{xc}}}{\delta n(\mathbf{r})} d \mathbf{r} \\
= & \mu^{\mathrm{KS}},
\end{aligned}
$$

where

$$
V^{\mathrm{KS}}(\mathbf{r})=V^{\mathrm{ext}}(\mathbf{r})+\int \frac{n\left(\mathbf{r}^{\prime}\right)}{\left|\mathbf{r}-\mathbf{r}^{\prime}\right|} d \mathbf{r}^{\prime}+\frac{\delta F_{\mathrm{xc}}}{\delta n(\mathbf{r})}
$$

is the Kohn-Sham potential.

In the zero-temperature theory, for a $(N+\eta)$-particle system $(\eta \rightarrow 0)$, the chemical potential $\mu^{+}$is located at the bottom of the conduction band whereas the chemical potential $\mu^{-}$for a $(N-\eta)$-particle system $\left(\eta \rightarrow 0^{\prime}\right)$ is located at the top of the valence band. Therefore we have 


$$
\lim _{\eta \rightarrow 0}\left(\lim _{T \rightarrow 0}\left(\mu^{N+\eta}-\mu^{N-\eta}\right)\right)=\mu^{+}-\mu^{-}=E_{g} \neq 0 .
$$

The discontinuity in the chemical potential leads to discontinuity in $V_{\mathrm{xc}}(0)$ at $T=0 \mathrm{~K} .{ }^{5}$ However, at finite temperature $T<<E_{g}$, the chemical potential will be located at the middle of the band gap for both the $(N+\eta)$ and $(N-\eta)$-electron systems in the limit $\eta \rightarrow 0$ [more precisely, the chemical potential will be located at the middle of the band gap when the number of thermally excited electrons (holes) $N_{e}\left(N_{h}\right)$ in the conduction (valence) band is much larger than $\eta$ ], i.e., we have

$$
\lim _{T \rightarrow 0}\left(\lim _{\eta \rightarrow 0} \mu^{N \pm \eta}\right)=\mu^{-}+\frac{1}{2} E_{g},
$$

so that there exists no discontinuity in the chemical potential at finite temperature. As a result, in the finitetemperature case, we expect also no discontinuity in the exchange-correlation potential $V_{\mathrm{xc}}(0)$ as $N+\eta$ crosses the band gap. Instead, from Eqs. (1) $-(4)$, we expect

$$
\begin{aligned}
\lim _{T \rightarrow 0}\left(\lim _{\eta \rightarrow 0} V_{\mathrm{xc}}^{N \pm \eta}(0)\right) & =\frac{1}{2}\left[V_{\mathrm{xc}}^{+}(0)+V_{\mathrm{xc}}^{-}(0)\right] \\
& =V_{\mathrm{xc}}^{-}(0)+\frac{\Delta}{2} .
\end{aligned}
$$

We shall show that such a result is indeed obtained from a finite-temperature generalization of our approximate theory developed in Ref. 10.

Our theory for $V_{\mathrm{xc}}(0)$ at $T=0$ can be generalized to finite temperature in a straightforward way. ${ }^{10,11}$ In the random-phase approximation (RPA), the following expression for $V_{\mathrm{xc}}(0)$ is obtained for a nonmagnetic system:

$$
V_{\mathrm{xc}}(0)=\left\langle\Sigma\left(\mathbf{k}, E_{k}\right)\right\rangle-\left\langle\int d \mathbf{r} V_{\mathrm{xc}}^{\mathrm{LDA}}(\mathbf{r}) \psi_{k}^{*}(\mathbf{r}) \psi_{k}(\mathbf{r})\right\rangle,
$$

where

$$
\begin{aligned}
\Sigma\left(\mathbf{k}, E_{k}\right)=\frac{1}{V} \int d \mathbf{r} \int & d \mathbf{r}^{\prime} \psi_{k}(\mathbf{r}) \psi_{k}^{*}\left(\mathbf{r}^{\prime}\right) \frac{1}{\beta} \\
& \times\left.\sum_{i \Omega_{n}} V_{\mathrm{eff}}^{\mathrm{RPA}}\left(\mathbf{r}-\mathbf{r}^{\prime}, i \Omega_{n}\right)\right|_{n=n(\mathbf{r})} \\
& \times g^{0}\left(\mathbf{r}^{\prime}, \mathbf{r}, E_{k}-i \Omega_{n}\right)
\end{aligned}
$$

is the RPA (Hedin $G W$ approximation) self-energy of the single-particle state $\psi_{k}(\mathbf{r})$ with energy $E_{k},{ }^{10}$ where

$$
\left.V_{\mathrm{eff}}^{\mathrm{RPA}}(q, \omega)\right|_{n}=\left.\frac{\boldsymbol{V}(q)}{\varepsilon(q, \omega)}\right|_{n},
$$

is the dynamically screened Coulomb interaction for a homogeneous electron gas with density $n$, evaluated at temperature $T$,

$$
g^{0}\left(\mathbf{r}, \mathbf{r}^{\prime}, i \varepsilon\right)=\sum_{k} \frac{\psi_{k}(\mathbf{r}) \psi_{k}^{*}\left(\mathbf{r}^{\prime}\right)}{i \varepsilon-E_{k}}
$$

is the one-particle Green's function and $V_{\mathrm{xc}}^{\mathrm{LDA}}(\mathbf{r})$ is the exchange-correlation potential evaluated at the localdensity approximation (LDA). Notice that $V_{\mathrm{xc}}^{\mathrm{LDA}}(\mathbf{r})$ and
$V_{\text {eff }}^{\mathrm{RPA}}(q, \omega)$ both change continuously across the band gap even at the zero-temperature limit. ${ }^{5,10,11}$ The average \langle\rangle in Eq. (13) is defined by

$$
\left\langle A_{\mathbf{k}}\right\rangle=\frac{\int d \mathbf{k} \frac{\partial f\left(E_{k}\right)}{\partial \mu} A_{\mathbf{k}}}{\int d \mathbf{k} \frac{\partial f\left(E_{k}\right)}{\partial \mu}},
$$

where $f$ is the Fermi distribution function and $\mu$ the chemical potential. At temperature $T \ll E_{g}, f(E)$ $\sim \exp [-\beta(E-\mu)]$ for states in the conduction band and $f(E) \sim 1-\exp [-\beta(E-\mu)]$ in the valence band. With this, it can be shown that the leading term in the smalltemperature expansion of Eq. (17) is given by

$$
\left\langle A_{\mathrm{k}}\right\rangle \sim\left[A^{\prime}\left(\varepsilon_{c}\right) N_{c}+A^{\prime}\left(\varepsilon_{v}\right) N_{h}\right] /\left[N_{c}+N_{h}\right],
$$

where

$$
A^{\prime}(\varepsilon)=\frac{\int d \mathbf{k} \delta\left(E_{\mathbf{k}}-\varepsilon\right) A_{\mathbf{k}}}{\int d \mathbf{k} \delta\left(E_{\mathbf{k}}-\varepsilon\right)} .
$$

$\varepsilon_{c}$ is the energy of the states at the bottom of the conduction band while $\varepsilon_{v}$ is the energy at the top of the valence band. $N_{c}$ and $N_{h}$ are the number of particles in the conduction band and number of holes in the valence band, respectively. With Eqs. (13)-(19), and using the fact that $N_{c} \rightarrow N_{h}$ as $\eta \rightarrow 0$, we obtain Eq. (12).

It is important to keep in mind that the absence of discontinuity in $V_{\mathrm{xc}}(0)$ in the $T \rightarrow 0, \eta \rightarrow 0$ limit does not imply that the correction $\Delta$ to the width of the band gap calculated in the Kohn-Sham theory vanishes. It simply means that the chemical potential is located at the middle of the band gap. A more interesting question to ask would be whether one can in fact determine from density-functional theory the change in the band gap as temperature changes. So far we have not found it to be possible. However, a simple analysis of the Dyson's equation within the RPA taking the band gap as the difference in the quasiparticle energy of the bottom state in the conduction band and the top state of the valence band suggests that the change in $E_{g}$ at low temperature is of order $\left(k_{B} T / D\right)^{m}$, where $D$ is the valence-band bandwidth and $m>1$. This correction is expected to be small at room temperature so that the zero-temperature theory should be reasonably applicable. (Of course, the band gap can be modified by other mechanisms such as phonons at finite temperature. These are not being considered in our present study.)

The author thanks M. Rasolt for directing him to the finite-temperature problem, and for helpful discussions. This work is supported by the National Science Foundation Grant No. DMR-85-21377. 
${ }^{1}$ L. J. Sham and M. Schlüter, Phys. Rev. Lett. 51, 1888 (1983). 2J. P. Perdew and M. Levy, Phys. Rev. Lett. 51, 1884 (1983).

${ }^{3}$ W. Kohn and L. J. Sham, Phys. Rev. 140, A1133 (1965).

${ }^{4} \mathrm{~W}$. Kohn and P. Vashishta, in Theory of Inhomogeneous Electron Gas, edited by S. Lundqvist and N. H. March (Plenum, New York, 1983), p. 79.

${ }^{5}$ L. J. Sham and M. Schlüter, Phys. Rev. B 32, 3883 (1985); see also L. J. Sham, Phys. Rev. B 32, 3876 (1985).
${ }^{6}$ R. W. Godby, M. Schlüter, and L. J. Sham, Phys. Rev. Lett. 56, 2415 (1986).

${ }^{7}$ N. D. Mermin, Phys. Rev. 137, A1441 (1965).

${ }^{8}$ T. C. Koopmans, Physica (Utrecht) 1, 104 (1933).

${ }^{9}$ See, for example, G. D. Mahan, Many-Particle Physics (Plenum, New York, 1981).

${ }^{10}$ T. K. Ng (unpublished).

${ }^{11}$ T. K. Ng and M. Hwang (unpublished). 